\title{
Visual outcome and ocular survival in pediatric ocular trauma
}

\author{
Lavanya Rao', Sulatha Bhandary², Krishna Rao Addoor ${ }^{2}$, Aditi Singh², \\ Suma Nair ${ }^{2}$, V.S. Binu ${ }^{3}$ \\ 'Department of Ophthalmology, Srinivasa Medical College, Suratkal, India; \\ ${ }^{2}$ Department of Ophthalmology, Manipal University, Manipal, India; ${ }^{3}$ Department \\ of Community Medicine, Manipal University, Manipal, India
}

\begin{abstract}
Purpose: Ocular injuries in children are a major under-recognized cause of ocular morbidity and can be prevented by identifying the etiology and implementing measures to prevent these injuries. This study intends to assess the visual outcome and ocular survival in pediatric ocular trauma.

Design: A descriptive study set in a tertiary care teaching hospital in Southern India.

Methods: A total of 56 children less than 18 years of age with ocular trauma during the period August 2010 to August 2012 were studied. Injuries were classified as per modified Ocular Trauma Classification System (OTCS). Final visual outcome was assessed at the end of one, three and six months.

Results: Of the 56 children assessed, $87.5 \%$ were boys. About $12 \%$ were under-fives and almost $66 \%$ were in the age range of 5-14 years. Majority (47.5 \%) had sustained trauma in the streets. Closed globe injury predominated (69.5\%). At presentation, grade I injury was noted in 28 eyes (47.5\%), grade II in one (1.7\%), grade III in three (5.1\%), grade IV in eight (13.5\%) and PL negative in three (5.1\%) eyes. Sixteen children were not cooperative for assessment of visual acuity (27.1\%).

At six months, good vision was noted in 44 (74.6\%), moderate in five (8.5\%), poor vision in one (1.7\%), PL negative in four (6.8\%) eyes and four children were un-cooperative (6.8\%). Four eyes had phthisis (6.8\%) and one eye (1.7\%) was eviscerated.

Conclusions: Ocular trauma in childhood was more common in the male child, mostly due to road traffic accidents. Visual acuity at presentation is one of the major criteria for the final visual outcome.
\end{abstract}

Key words: causes, ocular trauma, paediatric age group, preventive measures

\section{Introduction}

Eye injuries are a major under-recognized cause of disabling ocular morbidity affecting the young and are a leading cause of acquired unilateral blindness in childhood. ${ }^{1}$ However, nearly $90 \%$ of eye injuries can be prevented by relatively simple measures, although these may not always be possible. ${ }^{2}$ By identifying the factors in the etiology of these injuries, it may be possible to determine the most effective methods of reducing the incidence of visually damaging trauma. This study was carried out in order to assess the causes, demographic profile, visual outcome and complications of mechanical pediatric ocular trauma presenting to a tertiary care hospital.

Correspondence: Sulatha Bhandary, Department of Ophthalmology, Manipal University, Manipal 576104, Karnataka, India.

E-mail: sulatha bhandary@yahoo.co.in 


\section{Methods}

The study was carried out at the eye department of a tertiary care hospital, from August 2010 to August 2012. The study protocol was approved by the Ethics Committee of the hospital. A total of 56 children aged one to 17 years, treated for eye injury, formed the subjects of the study. At the time of presentation, name, age, gender, hospital number of the patients, mode of injury, time of injury, previous ocular complaints, place of injury, previous treatment if any were recorded. Cases of chemical injuries and trauma in previously diseased or injured eyes were excluded. Patients were divided into three age groups: pre-schoolers (one to four years), schoolchildren (five to 14 years) and adolescent (15 to 17 years).

Complete ophthalmic evaluation done at presentation included the evaluation of initial visual acuity, bony orbit, lids and adnexa, visual axis, ocular movements and anterior - posterior segment examination. Various methods of evaluating the vision included Snellen chart, random E s, Allen pictures, finger counting and assessment of light perception and projection of rays. For children too young to understand and cooperate with the above, vision was assessed by their ability to fix and follow light. Anterior segment evaluation included-location (zone) of the wound, presence of contusion, superficial foreign body, lamellar laceration, hyphema, relative afferent pupillary defect, presence or absence of cataract and subluxation or dislocation of lens. Posterior segment evaluation included - examination of the fundus for the detection of vitreous hemorrhage, retinal detachment, intraocular foreign body, signs of endophthalmitis and any other pathology. $X$ ray and CT scan of the orbit were done where necessary, as in cases of orbital fracture or suspected intraocular foreign body. The eyes were categorized according to 'The Ocular Trauma Classification system' with a modification to include two more categories-eyelids and orbital injuries. $^{3}$

All cases of open globe injury underwent primary corneal/corneoscleral tear repair, under general anaesthesia on an emergency basis. Patients were followedup over a period of one month, three months and six months depending upon the severity of injury. For minor injuries, one month follow-up was adequate. Final best corrected visual acuity and complications like phthisis, evisceration etcetera, if any, were noted at the end of the study period. The final visual acuity was graded according to the categories of visual impairment by the World Health Organization. In this study, vision greater than or equal to $6 / 18$ was taken as good visual outcome and vision less than 3/60 was considered as poor visual outcome. Vision in between these two categories was considered as moderate visual outcome. Ocular survival (i.e., preservation of the globe) was studied.

\section{Results}

Of the 56 children recruited into the study, 49 (87.5\%) were boys and seven $(12.5 \%)$ were girls; the total eyes evaluated were 59 since three children sustained injury to both eyes. Twenty-six children (44.1\%) encountered trauma to the right eye, 30 $(50.8 \%)$ to the left eye and three of them $(5.1 \%)$ to both eyes. 
The details of injuries are as follows:

The majority of injuries (66\%) occurred in the age group of 5-14 years and most of these occurred on the street (43.2\%). Almost all the injuries (85.7\%) among the pre-schoolers except one occurred at home. Details of these findings are depicted in Table 1.

Table 1. Age and place of injury of the children recruited into the study $(N=56$.)

\begin{tabular}{|c|c|c|c|}
\hline PLACE OF INJURY & $\begin{array}{c}\text { 0- } 4 \text { YEARS } \\
\text { N (\%) }\end{array}$ & $\begin{array}{c}\text { 5-14 YEARS } \\
\text { N (\%) }\end{array}$ & $\begin{array}{c}\text { 15-17 YEARS } \\
\mathbf{N}(\%)\end{array}$ \\
\hline HOME & $06(85.71)$ & $13(33.33)$ & $01(08.33)$ \\
\hline SCHOOL & 00 & $02(05.40)$ & 00 \\
\hline PLAYGROUND & 00 & $07(18.91)$ & $02(16.66)$ \\
\hline STREET & $01(14.29)$ & $17(43.58)$ & $10(76.92)$ \\
\hline
\end{tabular}

The time delay between sustaining the trauma and reporting to the hospital was as follows:

Twenty-five children (44.6\%) presented within six hours of injury to the hospital while another 14 (25\%) presented within 24 hours. However, it is of concern that about $30 \%$ of the children presented not earlier than after two days of the injury.

With respect to the type of injury, the observations were:

The most common kind of injury was blunt trauma (74.6\%) followed by penetrating trauma (18.6\%) and the remaining (5.1\%) were due to animal bites. One child with penetrating trauma had retained intraocular foreign body (IOFB) and the cause of injury was not known in one child who presented to us with a repaired corneal tear.

The most common cause of injury was found to be road traffic accidents (42.9\%) where children in the age group of 15 to 17 years were riding a motor bike/bicycle or were pillion riders. There were no reported gunshot injuries or those due to glass shards. Details of the injuries and the causes are illustrated in Table 2. 
Table 2. Nature and cause of injury among the study recruits $(\mathrm{N}=56$.

\begin{tabular}{|l|c|c|c|}
\hline & \multicolumn{3}{|c|}{ NATURE OF INJURY } \\
\hline CAUSE OF INJURY & $\begin{array}{c}\text { BLUNT } \\
\mathbf{n}(\%)\end{array}$ & $\begin{array}{c}\text { PENETRATING } \\
\mathbf{n}(\%)\end{array}$ & $\begin{array}{c}\text { IOFB } \\
\mathbf{n}(\%)\end{array}$ \\
\hline $\begin{array}{l}\text { Road traffic } \\
\text { accident (RTA) }\end{array}$ & $26(100)$ & 00 & 00 \\
\hline Sticks \& pencils & $1(25.0)$ & $2(50.0)$ & $1(25.0)$ \\
\hline Toys (bow \& arrow) & 00 & $1(100)$ & 00 \\
\hline Cans / stones & $08(100)$ & 00 & 00 \\
\hline Fireworks & $01(33.3)$ & $02(66.7)$ & 00 \\
\hline Sharp tools & 00 & $03(100)$ & 00 \\
\hline Falls & $08(88.9)$ & $01(11.1)$ & 00 \\
\hline $\begin{array}{l}\text { Animal \& bird } \\
\text { attacks }\end{array}$ & 00 & $01(25)$ & 00 \\
\hline Unknown & 00 & $01(100)$ & 00 \\
\hline
\end{tabular}

The injuries were typed applying the Modified Ocular Trauma Classification System and the majority (69.5\%) were observed to have closed-globe injuries, while another $20 \%$ were open-globe injuries. Four cases were restricted to trauma to the eyelids and another two had orbital injuries.

Visual acuity assessment at presentation revealed the grades of injury as follows: Of the 59 eyes, 28 eyes (47.5\%) (24 of closed globe, two of lid and orbit, respectively) had good vision (grade I), one moderate (grade II), 11 poor (grade III and IV) and three were blind (Grade V injury). Vision could not be assessed in sixteen children as they were uncooperative.

On correlating the grade of injury to the type of injury, it was found that all the patients with open-globe injury had a vision ranging from $6 / 30$ to no light perception, while $85 \%$ of those with closed-globe injury had good vision ( $\geq 6 / 12$ ) (Table 3 ). 
Table 3. Ocular features associated with blunt and penetrating trauma.

\begin{tabular}{|c|c|c|c|c|c|}
\hline & $\begin{array}{c}\text { Visual } \\
\text { acuity at } \\
\text { presentation }\end{array}$ & $\begin{array}{l}\text { Closed } \\
\text { globe } \\
\text { (no of } \\
\text { eyes) }\end{array}$ & $\begin{array}{l}\text { Open } \\
\text { globe } \\
\text { (no of } \\
\text { eyes) }\end{array}$ & $\begin{array}{l}\text { Lid } \\
\text { injury }\end{array}$ & $\begin{array}{l}\text { Orbital } \\
\text { injury }\end{array}$ \\
\hline \multirow[t]{6}{*}{ Grade of injury } & $\begin{array}{c}>6 / 12(20 / 40) \\
\text { grade I }\end{array}$ & 24 & 0 & 2 & 2 \\
\hline & $\begin{array}{l}6 / 15-6 / 30(20 / 50 \\
\text { to } 20 / 100) \text { grade II }\end{array}$ & 1 & 0 & 0 & 0 \\
\hline & $\begin{array}{c}<6 / 30 \text { to } 1.5 / 60 \\
(19 / 100-5 / 200) \\
\text { grade III }\end{array}$ & 1 & 2 & 0 & 0 \\
\hline & $\begin{array}{l}1.2 / 60 \text { to light } \\
\text { perception (LP) } \\
\text { Grade IV }\end{array}$ & 3 & 5 & 0 & 0 \\
\hline & No LP, Grade V & 1 & 2 & 0 & 0 \\
\hline & $\begin{array}{c}\text { No response } \\
\text { (not cooperative) }\end{array}$ & 11 & 3 & 2 & 0 \\
\hline \multirow[t]{3}{*}{ Zone } & I & $31(75.6 \%)$ & $8(66.7 \%)$ & - & - \\
\hline & II & $3(7.3 \%)$ & $3(25 \%)$ & & \\
\hline & III & $7(17.1 \%)$ & $1(8.3 \%)$ & & \\
\hline Pupil & RAPD + & 5 & 2 & 0 & 0 \\
\hline Cataract & & 0 & 3 & & \\
\hline Retinal detachment & & 0 & 03 & 0 & 0 \\
\hline Endophthalmitis & & 0 & 4 & 0 & 0 \\
\hline Hyphema & & 3 & 4 & 0 & 0 \\
\hline
\end{tabular}

NB 16 patients were uncooperative for vision assessment.

Patients with relative afferent pupillary defect (RAPD) were classified as pupil positive. Two children among the open-globe injury and five among closed-globe injury had RAPD.

Assessment of the zones of injury showed that among the open-globe type, $66.7 \%$ were in zone I (isolated to cornea) and $25 \%$ in zone II (corneo scleral limbus to a point five $\mathrm{mm}$ posterior into the sclera) and $8.3 \%$ in zone III (posterior to anterior five $\mathrm{mm}$ of the sclera). Among the closed-globe type $75.6 \%$ were in zone I (external), $7.31 \%$ in zone II (anterior segment) and $17.1 \%$ in zone III (posterior segment). 
The ocular features associated with trauma at presentation were as follows (Table 3): RAPD was noted in seven children, cataract in three, retinal detachment in three, endophthalmitis in four, IOFB in one and hyphema in seven. The three cases in which cataract was noted had penetrating injuries with respectively knife, bow-and-arrow and in one child, the cause was unknown. The three cases of retinal detachment were caused by blunt trauma secondary to a tennis ball injury, a cricket ball injury and a stone injury. All these three patients underwent retinal detachment surgery.

All four cases of endophthalmitis received intravitreal antibiotics $(0.1 \mathrm{ml}$ of 1.0 $\mathrm{mg}$ vancomycin and $0.1 \mathrm{ml}$ of $2.25 \mathrm{mg}$ ceftazidime) and two of them were culturepositive for Klebsiella and Microsporidia.

\section{The underlying cause of endophthalmitis in these four cases was as follows:}

In case one, the child had sustained trauma with a broom stick and presented with grade-IV injury with visual acuity of perception of light (PL) positive and projection of rays (PR) being inaccurate. This child presented to us 43 hours after the trauma and received intravitreal antibiotics on the day of admission. Here, the culture was positive for Klebsiella. However, after two days it progressed to panophthalmitis and was eviscerated immediately.

In case two, the trauma was with broom stick and presented with grade-IV injury with vision of PL-positive and PR-accurate. The child presented seven hours after the trauma. Two doses of intravitreal antibiotics were given 72 hours apart. However, on day seven the eye became PL-negative and on review at one month, the eye was phthisical.

In case three, the trauma was due to bow-and-arrow with grade-IV injury and presenting visual acuity of counting fingers at one meter. The child had intravitreal antibiotics and was discharged on day six with a visual acuity of hand movements. However, the child was PL-negative after six months and phthisical at eight months.

In case four, the child presented to us one week following trauma with a repaired corneal tear which was done outside. The visual acuity at presentation to us was hand movements positive. Child underwent pars plana vitrectomy with silicone oil injection and the visual acuity improved to $6 / 24$ at six month follow-up. The vitreous culture was positive for microsporidia.

Of the four cases, one underwent evisceration while the rest were subjected to vitrectomy; eventually however, two of the three eyes resulted in phthisis bulbi.

Of the seven cases that presented with hyphema, four had open-globe injury and three closed-globe injury. One patient had wooden IOFB, following a broom stick injury. The foreign body was removed at the time of primary repair but after one month the eye resulted in phthisis bulbi.

Follow-up was scheduled at the end of one, three and six months, depending on the severity of injury. Fifty-five children came for the first follow-up (post one month), 46 cases for the second (post three months) and another 46 cases came for the third follow-up, which was after six months. Visual acuity was assessed at each of these visits and the results are shown in Table 4. Forty-four cases complied with all three follow-ups, while one patient visited only once after six months. Eighteen 
patients with minor ocular injuries were advised only one follow-up visit at the end of one month. There appeared to be a significant improvement in the vision during the follow-up phase with $50 \%$ eyes demonstrating good vision at the end of six months (Table 4).

Table 4. Visual acuity at follow-up.

\begin{tabular}{|c|c|c|c|c|}
\hline VISUAL ACUITY & $\begin{array}{c}\text { BASELINE } \\
\mathbf{n}(\%)\end{array}$ & $\begin{array}{c}\text { 1 MONTH } \\
\mathbf{n}(\%)\end{array}$ & $\begin{array}{c}3 \text { MONTHS } \\
\mathbf{n}(\%)\end{array}$ & $\begin{array}{c}6 \text { MONTHS } \\
\mathbf{n}(\%)\end{array}$ \\
\hline Good & $28(47.5)$ & $36(62.1)$ & $30(65.2)$ & $31(67.4)$ \\
\hline Moderate & $01(1.7)$ & $06(10.3)$ & $04(8.7)$ & $04(8.7)$ \\
\hline Poor & $11(18.6)$ & $02(1.7)$ & $03(4.3)$ & $03(4.3)$ \\
\hline Blind & $03(5.1)$ & $05(8.6)$ & $03(4.3)$ & $04(8.7)$ \\
\hline Uncooperative & $16(27.1)$ & $09(15.5)$ & $06(13.1)$ & $04(8.7)$ \\
\hline TOTAL & $\mathbf{5 9}$ & $\mathbf{5 8}$ & $\mathbf{4 6}$ & $\mathbf{4 6}$ \\
\hline
\end{tabular}

The numbers of eyes with poor vision decreased over a period of time, from almost $19 \%$ at baseline to $7 \%$ at six months follow-up. However, the improvement appeared to plateau at the three months to six months follow-up period with no further improvement in the outcome.

Seven patients had poor vision at six months follow-up, four of whom had phthisis bulbi, one patient had undergone evisceration, one was post retinal detachment surgery with aphakia with best corrected vision of 3/60 and one more had significant irregular astigmatism due to corneal scarring (this child's parents refused further management).

There were four children with moderate vision during the final follow-up and they did not undergo amblyopia therapy. The details are as follows: one had injury due to fireworks and had multiple intra-corneal deposits and was advised penetrating keratoplasty. Two children with moderate visual outcome had sustained missile injury and had macular grade corneal opacity involving the pupillary area. The fourth child was injured by a stone and had central leucomatous grade corneal opacity which accounted for the moderate visual outcome.

\section{Discussion}

Eye injury in children is a menace and a common problem worldwide. In the present study, the maximum number of injuries was in the school-going age group of 5-14 years and the minimum in children under four years of age. This is similar to other studies done previously. ${ }^{4,5,9}$ Children under four years had lower incidence due to more protected and cocooned environment at home with their parents.

In this study, it was found that the male-to-female ratio was around 7:1. Many studies have shown that boys tend to be affected more commonly than girls, with male:female ratio varying from $2: 1$ to $4: 10^{6-8}$ This can be attributed to the greater 
physical activity and a more adventurous or aggressive nature of young boys during playing and interacting with their peer group.

The present study noted that the most common place of injury was in streets due to road traffic accidents (34\%). This is quite contrary to the results of other studies like those by MacEwen et al. and by Kaur and Agrawal, where home was the most common place of injury ( $45.6 \%$ and $51 \%$, respectively)., 4

In the above-mentioned studies, the most common place of injury was home and the injury at home was attributed to trauma with toys, sticks, stone, glass pieces and falls. In this study, home being the second most common place also emphasizes the fact that even home is not the safest place for young, active and curious children.

The most common mechanism of injury was blunt trauma (73\%). This is in accordance with previous studies by MacEwen et al. in Scotland (65\%) and other studies, but contrary to a study done in KGMU Lucknow (16\%), where penetrating trauma was more common (74\%). ${ }^{4,10}$

In this study, the most common cause of injury was found to be road traffic accidents (43\%). This can again be attributed to lenient traffic rules in this country. In other studies where penetrating injuries were more common, injury due to stick, wood etcetera, while playing were more common probably due to the rural setting of these studies. ${ }^{9}$ In the present study, three children had trauma while playing with a knife unsupervised at home.

Other studies found high prevalence of ocular trauma in sport-related activity (MacEwen et al., Grin et al.) like baseball, tennis and basketball., 411 In the present study, $13 \%$ of children had ocular injury due to cricket and tennis balls.

Uncommon causes of injury in this study were trauma with bird's beak and dog bite. Two children had injury due to fireworks and both became PL-negative. None of the children in this study sustained trauma due to gunshot.

It is important that the injured follow-up at least for the first three months as this is a period of remarkable improvement in vision as depicted in the study. Visual acuity at presentation is one of the major criteria of visual outcome in cases of trauma. In open-globe injury, seven children had poor vision at presentation and six of them had poor final visual outcome. Only one child with poor vision at presentation improved to moderate visual outcome at six months follow-up. In closed globe, 24 children had grade-I injury and continued to have good vision at the final follow-up. One child with grade-V injury at presentation had no perception of light during the final follow-up. Hence the results of this study also confirm that the visual acuity at presentation is one of the major criteria predicting the final visual outcome.

Adequate steps should be taken to prevent ocular trauma in children. Prevention of injury depends, firstly, on identifying the cause and, secondly, targeting the cause by education and implementation of legislation.

Common household articles such as broom sticks, knives and wooden sticks can prove to be dangerous for the child's eye as is seen in the study. The home environment should therefore be made child-friendly by keeping sharp objects out 
of reach of children and padding the corners of couches, tables and chairs. Children should be kept away from dangerous toys and handling of fireworks. Moreover, there is an urgent need to implement strict traffic rules. Wide publicity with regards to these aspects could be a way forward. The need for compliance with follow-up also needs to be stressed to the parents of injured children.

\section{References}

1. Parver LM. Eye trauma: The neglected disorder. Arch Ophthalmol1986;104:1452-1453.

2. Moreira CA, Debert-Ribeiro M, Belfort R. Epidemiological study of eye injuries in Brazilian children. Arch Ophthalmol 1988;106:781-784.

3. Pieramici DJ, Sternberg P, Aaberg TM, et al. A system for classifying mechanical injuries of the eye (globe). The Ocular Trauma Classification Group. Am J Ophthalmol1997;123:820-831.

4. MacEwen CJ, Baines PS, Desai P. Eye injuries in children: The current picture. Br J Ophthalmol 1999;83:933-936.

5. Strahlman E, Elman M, Daub E, Baker S. Causes of pediatric eye injuries. A population based study. Arch Ophthalmol 1990;108:603-606.

6. Diniz MC, Tzelikis PFM, Alvin HS, et al. Trauma ocular em criança abaixo de 15 anos: prevenção baseadaem estatísticas. Rev Bras Oftalmol 2003;62:96-101.

7. LaRoche GR, Mclntyre L, Schertzer RM. Epidemiology of severe eye injuries in childhood. Ophthalmology 1988;95:1603-1607.

8. Silva RE, Nassaralla BRA. Prevalência de trauma ocular infantil no ambulatório de SUS do Instituto de olhos de Goiânia. Rev Bras Oftalmol 1998;57:865-868.

9. Apjit K, Ajai A. Paediatric ocular trauma. Curr Sci 2005;89:43-46.

10. Cariello AJ, Moraes NS, Mitne S, et al. Epidemiological findings of ocular trauma in childhood. Arq Bras Oftalmol 2007;70:271-275.

11. Grin TR, Nelson LB, Jeffers JB. Eye injuries in childhood. Pediatrics1987;80:13-17. 